\title{
CLINICAL IMPLICATIONS OF CHILDHOOD BILINGUALISM
}

\author{
Frenette Southwood, Stellenbosch University
}

\section{INTRODUCTION}

In many parts of the world, bilingualism ${ }^{1}$ is the norm rather than the exception. South Africa, with its 11 official languages, is one of these parts. Already in 1992, Stobbart (1992:23) stated that speech-language therapists (henceforth "clinicians") in South Africa need to become aware of the implications of service delivery in a multilingual and multicultural set-up. According to Grosjean (1989:14), "we should always keep in mind that half the world's population is bilingual and that using the monolingual as a yardstick is questionable". It would appear, on the one hand, that clinicians' sensitivity toward and knowledge of issues pertaining to bilingualism and biculturalism are growing. On the other hand, however, their approach to the assessment and remediation of bilingual clients remains inherently monolingual $^{2}$, as does their clinical training (Müller 2003:2).

In this article, clinical implications of childhood bilingualism ${ }^{3}$ for assessment instruments and assessment methods, as well as for therapy provision, will be dealt with. Thereafter, brief comments will be made on the use of translators and interpreters during assessment and/or therapy.

\section{ASSESSMENT}

The purpose of bilingual assessment, as stated by Damico (1991:158), is as follows:

The evaluation [of the bilingual child's communication abilities - FS] should differentiate between difficulties that result from normal second-language-learning processes or differences due to experiential and cultural factors versus those caused by intrinsic language-learning impairment. 
To differentiate between the two, the clinician needs to establish whether the client has difficulties in both languages (which would point to a language impairment) or only in the second language (which would point to normal second languagelearning difficulties). Because a communication disorder only exists if it is evident in both languages, it is necessary to assess both languages for diagnostic purposes.

In this section, the implications of childhood bilingualism for various aspects of assessment, namely case-history-taking, formal and informal assessment, and test interpretation, will be discussed. Suggestions that have been made for more appropriate assessment of bilingual clients will also be discussed. When considering the assessment of bilingual clients, the following seemingly obvious statement needs to be kept in mind: A clinician can only assess those languages of her client that the clinician herself speaks with a the level of proficiency equal to or close to that of a first language speaker. If the clinician does not know ${ }^{4}$ one of the languages of her client, then she cannot possibly assess that language, except through an interpreter. Where clinicians do not know all the languages of bilingual clients, the completion of the assessments of these clients often has to be spread over several individuals, each competent in one of the client's languages (Müller 2003:6). Although this practice delivers valuable information on each of the client's languages, it does not provide any information on phenomena that are typical of the communicative behavior of bilinguals, such as lexical borrowing, code switching, and interference, and it fragments the evaluation process.

\subsection{The case-history}

Clinicians generally agree that a thorough case-history is one of the most powerful diagnostic and therapy planning tools. Through case-history-taking, the clinician seeks to (i) establish possible etiological factors; (ii) identify factors or occurrences in the client's language environment in home, school, and other settings that may positively or negatively affect the speech or language problems and/or may contribute to the maintenance of these problems;

(iii) gain information that might be useful during therapy planning and activity selection; and (iv) determine the prognosis. 
Many clinicians have a standard case-history form that they routinely complete while interviewing clients suspected of having communication difficulties, or the caregivers of such clients, regardless of the client's language background. Where the client is a child, this standard case-history typically covers areas such as biographical information, medical history (including pregnancy and birth information), developmental history and milestones (motor, sensory, and social), and educational information. Detailed answers to questions regarding the home environment (including child-care arrangements, and social, psychological, or other dysfunctions of members of the household), child-rearing practices, and attitudes toward physical, mental, or sensory disabilities are often not obtained. Answers to these questions must, however, be obtained if the clinician seeks to improve the chances of making an accurate diagnosis, setting realistic goals for therapy, selecting appropriate therapy activities, drawing up an effective home programme, and establishing a realistic prognosis.

One implication of treating a bilingual and, therefore, possibly bicultural client is that the clinician needs to modify the case-history format to include questions on matters not usually inquired about from a client who shares the clinician's language and cultural background ${ }^{5}$.

In many cases, the clinician may share a variety or dialect of one language with the bilingual client, but may not necessarily share one of the client's cultures. The clinician should guard against assuming that her cultural values hold for her client, even if a variety of (one of) her language(s) is spoken by the client. According to Miller (1984a:169), it is necessary to be aware of normal variations in patterns of upbringing, socialisation, and attitudes (especially attitudes toward and beliefs about handicapping conditions - Harris 1986:234) in order to gain a reliable picture of clients from different cultural and language traditions ${ }^{6}$. Therefore, clinicians should aim to acquaint themselves with the beliefs and practices of the community which they serve ${ }^{7}$.

One might claim that there is always a culture clash of some degree between any client's normal daily activities and the clinical encounter consisting of referral, assessment, therapy, and eventual discharge from speech-language therapy (Müller 2003:4). This clash may, however, be more noticeable where the clients involved in this encounter are from backgrounds different to that of the majority of the health professionals. For most middle- 
class, Western clinicians, the system of general practitioner referrals, special tests, and detailed interviews by various health professionals may make sense, but to some clients (for example, those used to having their medical problems attended to by the familiar local health worker), finding themselves being "processed" in this way may be an daunting experience. The families of clients that arrive with their children for a speech-language assessment may already have surmounted numerous bureaucratic and intercultural communication hurdles by the time they reach the clinician. As Miller (1984a:173) suggests, there may be dissatisfaction with how they have been handled to date, and there may also be confused impressions as to why they are there and what will be done. Furthermore, families of all cultures may be reluctant to admit the presence of handicaps in other relatives because of a loss of prestige that would affect the family at large if the truth were known (Miller 1984a:172). Similarly, families may be hesitant or unwilling to share information regarding certain forms of social behavior $^{8}$. For these reasons, certain mental or physical disabilities and family background information may be kept hidden even if direct questions are asked about them. It is in this context that the case-history is taken.

In order to improve the possibility of obtaining an accurate case-history (from clients of all culture groups, but especially from those groups, whether bilingual or monolingual, with which the clinician is not well-acquainted), the clinician needs to practice good interviewer skills ${ }^{9}$. According to Schreier (1991:351) and Westby (1990), these skills include the following:

(i) Using open-ended questions most of the time, especially initially. Examples of openended questions include "Why are you here today?", "What do you want me to do for you?", "What, according to you, is wrong with (the client)?", and "Tell me about the languages you use at home".

(ii) Using brief, simple questions that are not negatively phrased. For example, the negatively phrased "(The client) is not showing signs of hearing difficulty, is he/she?" may elicit "yes", meaning "yes, he is" or "yes, that is right, he is not", or it may elicit "no", meaning "no, he is not" or "no, that is not right, he is" ${ }^{10}$.

(iii) Using direct questions if responses to open-ended questions need elucidation.

(iv) Attempting to detect clues regarding hidden agendas. The clinician needs to establish whether the caregiver is using an apparent problem with the client to gain help with a 
certain domestic situation (such as child abuse, emotional abuse of family members, or marital problems), especially when the topic of domestic problems is taboo in the caregiver's culture(s). According to Schreier (1991:351-352), caregivers may be reluctant or hesitant to touch on these subjects but may nevertheless hope that the interviewer will "guess" what they are hiding and offer an avenue of helpful intervention.

For bilingual clients, it is strongly recommended that the format of the case-history be changed to include very specific questions regarding language use, in order to reveal not only what languages the client uses, but also their pattern of use. Instead of relying solely on caregiver statements such as "He speaks English and Afrikaans, with Afrikaans being his better language", a profile of the client's language use should be drawn up in order to establish what language the client speaks to whom, where, when, how, and possibly also why ${ }^{11}$. Questions on the following should be included: the sequence of acquisition of the languages (simultaneous or sequential), the client's communication network (including the language(s) of tuition at the educational institution), and the value attached by the family to each language.

If the clinician, instead of the caregiver, draws up the profile, the information obtained may be incomplete, and, therefore, less useful if care is not taken by the clinician to ensure that the nature of interactions are sampled representatively before drawing up the profile, that the setting(s) in which these interactions take place are natural to the client and to the conversational partner, and that the methods of recording the client's language behaviour are appropriate (Miller 1984b:21-22).

\section{$2.2 \quad$ Formal assessment}

Formal assessment instruments use normative data to compare children with their peers (Toronto and Merrill 1983:107). Although the administration of formal, standardised tests does have its advantages ${ }^{12}$, there are problems with the fairness and interpretation of these tests when performed on monolinguals ${ }^{13}$. These problems also hold when the tests are performed on bilingual clients, but when the client is bilingual and also possibly bicultural, 
additional concerns exist. Most clinicians are aware of the inappropriateness of administering tests on bilinguals when these tests have been standardised on monolinguals. Clinicians' usual responses to this inappropriateness are to (i) translate the test; (ii) remove or replace "problem" vocabulary items or "problem" syntactic structures; and/or (iii) expand profiles to include questions, items, or categories relevant to the local bilingual population. As will be shown below, each of these responses is unsatisfactory to a certain degree.

Test translation, while tempting, is not an ideal solution to the problem of the unavailability of appropriate assessment material for bilinguals. One reason for this is that translating items can cause changes in the level of difficulty of items (Peña, Quinn and Iglesias 1992:269) (e.g., He doesn't feel like eating may or may not be simpler than its Afrikaans counterpart Hy is nie lus om te eet nie). In addition, kinship terms, colour terminology, attitudes toward the roles of different family members, and the role of animals as pets and food may differ among cultures $^{14}$ (Miller 1984b:112-113). These are areas frequently covered in formal language tests for children. Furthermore, still only those structures or words deemed important in the original test language will be assessed, and some of these structures or words may even be lost in translation (Erickson and Iglesias 1986:204). Lastly, translation negates the use of any of the norms established in the original test language (Erickson and Iglesias 1986:203).

The removal or replacement of "problem" items may be a solution, but, again, it must be noted that modifying an assessment instrument negates the use of any of the norms established for the original instrument. Such removal or replacement can, furthermore, only be done by someone with a thorough knowledge of the language and cultural background of the intended test population ${ }^{15}$.

When expanding profiles to include questions, items, or categories relevant to the local bilingual population, one requires norms of the language behavior of this local bilingual population. Without norms or criteria, the profiles will not be interpretable.

Test-internal factors such as those mentioned above (the language of the assessment instrument and the culture-boundedness of some test items) can influence formal assessment, but so can test-external factors, such as the attitude and experiences of a language community 
toward formal assessment (Peña and Quinn 1997:324). Language assessment does not take place in a cultural vacuum. A contrast in attitudes toward test-taking and/or in response styles of the clinician and the client may exist. Clinicians and educators often uphold values such as try-your-hardest, answer-at-all-costs, and work-hard-and-it-will-get-you-somewheresomeday. Some cultures tend to encourage values different to these. For example, according to Miller (1984c:109), some cultures may "frown upon individualism as disrespect for the wisdom of elders or the collective decision-making role of the family or community group. Similarly, striving to emulate one's peers in competition might be taken as betraying the communal spirit of mutual support and achievement of goals through common effort". According to Chamberlain and Medinos-Landurand (1991:118), the extent to which competition is valued in a culture may influence the client's performance on tests.

Given these test-internal and test-external factors that may lead to unfair assessment of bilingual clients' language abilities, Erickson and Iglesias (1986:200-201) recommend that standardised assessment instruments be administered in a nonstandard manner. This may provide the bilingual client with the opportunity to perform at the maximum level of his ability, i.e., it may improve the chances of truly establishing what the client can and cannot do. The following nonstandard practices regarding test administration are recommended by Chamberlain and Medinos-Landurand (1991:132-136) and by Erickson and Iglesias (1986:200-201):

(i) The examination of each item before administering the test. The clinician should decide whether or not the client has had exposure or access to what is being assessed $^{16}$.

(ii) The rewording of instructions, using simpler syntactic constructions than those prescribed by the manual, or giving the instructions in the language or language variety in which the client is reported to be most proficient.

(iii) The provision of additional time for the client to respond to tasks during testing.

(iv) The continuation of testing beyond the ceiling. The information obtained by doing so may be very valuable when planning therapy.

(v) The recording of all responses, particularly when the client changes an answer, explains, comments, or demonstrates using gestures. 
(vi) The comparison of the client's answers to (a) the language varieties that he is exposed to or (b) first and second languagelearning features, after which articulation and expressive language samples should be rescored, giving credit for variation or differences.

(vii) The development of several practice items so that the process of test-taking is established, should the test not already have a substantial number of such items.

(viii) Having the client name the picture in addition to pointing to the stimulus item to ascertain the appropriateness of the label for the pictorial presentation when administering picture vocabulary recognition tests ${ }^{17}$.

(ix) Having the client explain why he selected the answer that is incorrect according to the manual ${ }^{18}$.

(x) Having the client identify the actual object, body part, action, photograph, etc., particularly if he has had limited experience with books, line drawings, or the assessment procedure.

In the literature, there is a longstanding discussion on the necessity of bilingual assessment as opposed to double monolingual assessment, as the monolingual approach to assessment leads to lower scores for bilinguals. Bilingual assessment entails not treating the client as if he is merely two monolinguals in one body, i.e., not assessing one language first and comparing the results to those of first language speakers and then doing the same for the other language, but rather using assessment instruments that take into account both the clients languages at once. In the light of this, South African clinicians cannot assess their bilingual clients adequately in a formal manner, because no such bilingual assessment instruments are available.

\subsection{Informal assessment}

According to Toronto and Merrill (1983:107), informal assessment instruments determine individual strengths and weaknesses for possible remedial recommendations. Miller (1984b:24) states that informal assessment does not replace standardised testing in bilingual settings but is an important adjunct to such tests. Informal assessment often provides the clinician with a fair idea of what areas require attention during formal assessment, should it be possible to perform such formal assessment. The use of nonstandardised profiles, language 
samples, developmental charts and scales, questionnaires, and diaries are examples of methods of assessing a client's language in an informal manner to obtain information that supplements the results of standardised testing. For many language groups, especially minority ones, there are, however, very few standardised assessment instruments available, and those that are available often lack cross-cultural validity (Craig and Washington, 2000:366; Toronto and Merrill, 1983:105). In these cases, informal assessment does replace standardised testing.

In the absence of formal assessment instruments for expressive language abilities, the clinician often has to rely on language sample analysis. In the case of the bilingual client, it is more important than ever to obtain more than one language sample with more than one communication partner. Bilingual clients may or may not, for example, code switch in a particular situation and with a particular conversation partner. Therefore, a variety of language samples will increase the chances of obtaining a representative picture of the client's expressive language abilities. However, even if representative samples are obtained, the clinician cannot compare the performance of the client with that of his peers without normative data. This means that the clinician may not be able to judge the sample qualitatively due to a lack of normative data for the language and/or cultural group to which the client belongs. In these cases, the clinician can use typical bilingual developmental trends as a guide, bearing in mind, for example, what is to be expected of any bilingual child who has had the amount and quality of exposure to languages that the client has had. Therefore, even without access to norms, the clinician can determine whether the client follows the common developmental patterns of bilinguals and can compare the client's proficiency in one language to that in the other.

Developmental charts and scales are to be used with caution, because developmental norms may vary among culture (and, therefore, language) groups, with the question of "what is normal when?" not receiving the same answer for every group ${ }^{19}$. Charts and scales developed for monolingual, monocultural groups should, therefore, not be applied "as is" to bilingual clients. 
A questionnaire is often seen as more structured than a profile, chart, or scale and may, therefore, be more easily completed by the caregiver. Unfortunately, questionnaires are often unreliable, among other shortcomings, due to the fact that people respond in the way they think the clinician wants them to respond (Miller 1984b:23). This last point also applies to diaries kept by caregivers.

Despite the bleak picture painted thus far in this section, some encouragement is to be taken from the work done by the University of Massachusetts, Amherst's NIH Working Groups on African American English, who developed and standardised a screening and a diagnostic test to distinguish difference from disorder in the language of African American English-speaking children (cf. Seymour, Roeper, and De Villiers 2003a, 2003b; De Villiers, De Villiers, Roeper, Seymour, and Zurer Pearson 2003). Encouragement should also be taken from the work of Craig and Washington and their colleagues on determining culturally fair means of assessment of African American English-speaking children ${ }^{20}$ (cf, e.g., Craig and Washington 1994; Craig, Washington, and Thompson-Porter 1998a, 1998b; Washington and Craig 1998, 1999). They propose the use of measures such as mean length of communication (in words and in morphemes), number of different words used in a sample consisting of 50 communication units, presence of complex syntax, comprehension of requests for information, and comprehension of active/passive sentences when assessing the language abilities of these children. Craig and Washington (2000) found that these five measures successfully differentiated between those African American English speakers who were language-impaired and those who exhibited typical language development. In order for these measures to become part of the clinical practice of those clinicians with African American English-speaking clients, norms now need to be obtained for each of these five measures.

As in North America, the scarcity of normative data also impedes informal assessment of bilingual clients in South Africa. Developmental norms can, however, not be developed for all bilingual South Africans. Because of the multiple languages spoken in the country and because no two bilingual clients share the same amount, length, and quality of exposure to their two languages, it will not be possible to compare bilinguals to each other for the purposes of norm-referenced testing (Jordaan and Yelland 2003:28). Rather, the respective 
abilities in each language will have to be considered in relation to the amount and type of input received in each of the languages (Jordaan 1996:353).

\section{$2.4 \quad$ Test result interpretation}

Taylor (1986:13) proposes the following revision of Van Riper's definition of communication disorders:

Communicative behavior by an individual can only be considered defective if it deviates sufficiently from the norms, expectations, and definitions of his or her indigenous culture (or language group); that is, if it is (a) considered to be defective by the indigenous culture or language group, (b) operates outside the minimal norms of acceptability of that culture or language group, (c) interferes with communication within the indigenous culture or language group, (d) calls attention to itself within the indigenous culture or language group, or (e) causes the user to be "maladjusted" as defined by the indigenous culture [or language group - FS].

This definition clearly implies that the clinician needs to have knowledge of both the client's languages and normative data for the client's language and cultural group(s). Without this knowledge, the clinician cannot decide whether or not the client has communication problems. Should the clinician know the client's languages, and cultural systems and beliefs, she needs to consider the following before concluding that the client with limited proficiency in a language is indeed language-impaired, and is not merely in the normal process of second languageacquisition (Langdon 1989:160-162):

(i) The exposure to the language: Has it been limited or not? Has it been interrupted for significant lengths of time? Did the exposure come from first language speakers of the language?

(ii) Length of school attendance: Has the client had enough opportunity to develop proficiency in using the language for academic purposes (Cummins's "cognitive/academic language proficiency" or "CALP") or is the client still in the process of trying to learn general conversational skills in the language (Cummins's "basic interpersonal communication skills" or "BICS") $)^{21}$ ? 
(iii) Uses of language: Does the client really have limited proficiency or is he simply not responding as one would expect a monolingual middle-class child to respond? That is, are there any cultural or sociolinguistic factors at play?

(iv) Peer comparisons: How does the client fare when compared to other children from the same background with similar language experiences?

(v) Health and developmental factors: Have mental retardation, malnutrition, emotional handicaps, and physical impairments (motor, visual, and/or hearing) been ruled out?

Stockman (1986:118) states,

notions of normalcy influence every clinical decision made about speakers who exhibit language impairment. Clinical judgements about atypical language behavior must be guided by some explicit or implicit definition of what constitutes normal behavior. Likewise, the formulation of therapy goals and the evaluation of treatment progress are guided by assumptions about the requirements for normal language performance.

The absence of acquisition data (i.e., normative data) is a barrier to the fair and accurate language assessment of bilingual speakers.

Two main problems can be identified with the interpretation of test results of bilingual clients. The first is overdiagnosis (where the client is diagnosed with a communication disorder despite his communication abilities being normal) and the second is underdiagnosis (where the client has a communication disorder, but is not diagnosed as such). The possible effect of underdiagnosis on the client's future communicative abilities and educational progress is obvious, whereas Müller (2003:5) states that over-referral (and, therefore, by implication, overdiagnosis) not only labels clients with stigmatised conditions, but also drains often underfunded and/or undersupplied services ${ }^{22}$.

There are three reasons for errors of overdiagnosis of bilingual clients. Firstly, the client may be identified as having a communication disorder through the use of an assessment instrument standardised on a population other than the client's own. Secondly, the client who speaks a 
nonstandard variety of a language may be diagnosed as having a communication disorder by an assessment instrument standardised on a group of which only a subgroup consists of speakers of a nonstandard variety. Thirdly, there may be a lack of norms or criteria by which to judge the bilingual child's language performance. As stated by Gutiérrez-Clellen, Restrepo, Bedore, Peña, and Anderson (2000:89), "without basic language criteria, clinicians may underestimate the language skills of bilingual children".

There are two reasons why underdiagnosis may occur. The first is that the clinician is unaware of the specific ways in which the client's language variety differs systematically from the standard variety of that language and, therefore, she attributes any variance in the client's linguistic system to differences between the client's language variety and her own (or the standard) variety of the language, possibly missing the presence of pathology. Secondly, the clinician may be overly wary of treating bilingual clients in a way that could imply prejudice on her part (especially if the clients are from a culture different to hers).

When interpreting assessment results for caregivers, caution should be exercised in the manner in which information is conveyed. To be told to come back after some weeks or months have passed may be interpreted as being handled in an uncaring or incompetent manner (according to Miller 1984a:172). An explanation should be provided as to why no immediate intervention is indicated. Referrals to other professionals, such as to audiologists or pediatricians, may confuse people who are used to receiving all their health-related treatment from one person (Miller 1984a:172). Therefore, care should be taken to explain the need for and relevance of such referrals. As in the case of monolingual clients, the clinician should carry over the assessment interpretation to the caregivers of bilingual clients in such a way that they see the sense in having attended the assessment session, and are able to confidently explain the outcome of the session and the reason for further referrals to those interested household members who did not attend the session.

\subsection{Problems with assessment: What are the options?}

This section aims to summarise the problems with assessment, and the solutions offered, discussed thus far. Already in 1983, Vaughn-Cooke (1983:29-33) proposed a range of 
alternatives to traditional, inappropriate assessment instruments ${ }^{23}$, which are listed below. Unfortunately, as will be seen, each of these proposals is in some way problematic.

(i) Standardise existing tests on nonmainstream speakers. At first sight, this appears to be a reasonable proposal, as the cost of test development is spared, and clinicians' "favourites" (tests that clinicians find easy and quick to administer, as well as valid) can be standardised for clients from other language and cultural groups. However, according to Peña and Quinn (1997:324-325), there are several problems with the practice of obtaining local norms for assessment instruments for bilinguals. One problem is that bilinguals do not form a heterogeneous population. Apart from the actual language combinations that differ, bilinguals themselves differ in terms of, among others, the amount of exposure to each language, the quality of the input received, the age of first exposure to each language, the sequence of exposure to the languages, and the rate of acquisition of the languages. Because of these variations in bilingual acquisition patterns, it is not possible to obtain one set of norms for all bilingual language acquisition. Another problem with obtaining local norms is that this practice often results in lower norms for the bilingual speaker when compared to the original normative population, as the assessment instrument was designed with the monolingual population in mind. Lower norms may be seen as indicative of lower potential. At best, lower norms for bilinguals may lead to lower expectations for the bilinguals; at worst, lower norms for bilinguals, especially if most of these bilinguals belong to the same ethnic groups, may imply genetic inferiority of these groups. A third problem with re-norming standard language tests is that these tests can only reveal what the speaker of a nonstandard variety of the language knows about the standard variety of the language. Lastly, simply because norms have been obtained, it does not mean that the test is then appropriate for the bilingual client.

(ii) Include a small percentage of nonmainstream speakers in the standardisation sample when developing a test. The first problem with this proposal is that, due to the high cost, new South African tests are not likely to be developed frequently. This proposal does not solve problems with existing tests that are available to the clinician. The second problem is that it is not clear exactly who this small percentage of speakers is supposed to represent. 
(iii) Modify or revise existing tests in ways that will make them appropriate for bilingual or bidialectal speakers. Here it is important that the person revising the test has a thorough knowledge of the languages or dialects involved before initiating revisions. The problem with revisions is the required re-standardisation, which could be a lengthy and costly process.

(iv) Utilise a language sample when assessing the language of bilingual speakers. The problem with this practice is that the results from such analyses must be interpreted within a developmental framework. Unless such a framework exists for the language variety of the client, the language sample will not be fully interpretable, and this option (on its own) will not be sensible.

(v) Utilise criterion-referenced measures when assessing the language of bilingual speakers. The question here concerns whose criteria should be used. As with language samples, developmental data are needed for interpretation.

(vi) Refrain from using any standardised tests that have not been corrected for test bias when assessing the language of bilingual speakers. Although this would probably be the fairest (short-term) solution, the question arises as to what the clinician can then use.

(vii) Develop a new test which can provide a more appropriate assessment of the language of bilingual speakers. If one wants to assess the language of these speakers formally, then this is theoretically the best option. Unfortunately, it is neither practical nor costeffective in the South African situation, and it sheds no light on what clinicians should do in the interim. Also, due to the immense diversity in the South African clinical population, these new language appropriate and culturally appropriate tests are almost certainly in their turn bound to be used on populations for which they were never intended.

In the light of the above discussion, it appears that alternative approaches to language assessment should be considered. The identification of pragmatic difficulties instead of surface difficulties has been proposed as one such alternative approach ${ }^{24}$, where pragmatic difficulties are evidenced in, for example, (i) linguistic nonfluencies such as unusual hesitations, unexpected pauses, and repetitions; (ii) frequent revisions; (iii) delayed responses at turn-switching points; (iv) the need for multiple repetitions before comprehension occurs; 
(v) the use of nonspecific referring terms such as that, there, and then, or he, where it is not clear what is being referred to; (v) poor topic maintenance; and (vii) inappropriate responses, and where surface difficulties are evidenced in errors of morphology and/or syntax. Another alternative is the use of dynamic assessment, where the client's response to therapy is taken into account through a test-teach-retest design in assessment, before deciding whether the client is language-impaired or not. Peña et al. (1992) found that this assessment approach successfully differentiated nondisabled American children of Puerto Rican and AfricanAmerican descent from their peers who were language-impaired. Lastly, Rollins, McCabe, and Bliss (2000:226) recommend the assessment of the client's personal narratives by using story prompts ${ }^{25}$, instead of assessing his surface language difficulties, as this method of assessment has been shown to be appropriate for use with clients from a variety of cultural backgrounds.

\section{THERAPY}

Childhood bilingualism does not have implications for assessment only, but also for therapy. In this section, these implications for choice of language during therapy, for parent guidance, and for therapy in the school setting will be discussed.

\subsection{Language choice in therapy}

The choice of language(s) or language variety is a critical aspect in clinical intervention in bilinguals (Müller 2003:8). According to Miller (1984a:183), the initial aim of therapy should be to develop communicative abilities in keeping with mental age in at least one of the client's languages. Miller and Abudarham (1984) agree, stating that it is advisable that the clinician concentrate on one language at a time. Based on their research results ${ }^{26}$, Perozzi and Sanchez (1992:350, 351) state that this "one language" should be the client's first language.

The practice of first instructing typically-developing bilingual children in their first language before instruction in their second language commences is well-established (Perozzi and Sanchez 1992:348). This approach is based on the interdependence hypothesis of Cummins (1984, 1989), also called the "common underlying proficiency hypothesis". This hypothesis 
claims that experience with either language can promote development of the proficiency underlying both languages ${ }^{27}$, given adequate motivation and exposure to both languages; to the extent that instruction in the one language succeeds in increasing proficiency in that language, transfer of such proficiency to the other language will occur. In other words, at least one of a person's languages must be developed to a certain level in order for the person to benefit fully from instruction in another language. Although Cummins's work was on the development of literacy and other academic skills in typically-developing minority-language children, his research results have been applied in the fields of remedial teaching and speechlanguage therapy.

Not all scholars agree with Miller and Abudarham's (1984) recommendation of concentrating on one language at a time (cf., e.g., Gutierrez-Clellen 1999; Thordardottir, Weismer, and Smith 1997). Despite the criticisms levelled against the one-language approach, this is the approach that many South African clinicians will have to follow, because it may not be possible for a clinician to provide therapy in more than one of the client's languages: either because the clinician is monolingual or because, despite being bilingual, she is not proficient enough in the client's other language(s) to provide therapy in that language. In these cases, the language in which the client requires therapy needs to be determined, because, if that language is not one that she can provide therapy in, the client will have to be referred to another clinician.

Another case in which a decision on the language of therapy is required, is where the client is what Valdés and Figueroa (1994) call a "circumstantial bilingual", i.e., a person who has both his languages as first language. If the clinician wants to follow the one-language approach with these clients, it needs to be decided in which language the client should receive therapy first. As mentioned above, it is the view of Perozzi and Sanchez (1992) that bilingual children should receive intervention in their first language. When the clinician is not proficient in both or all the client's languages and when the client is a circumstantial bilingual, factors other than the client's first language should be considered when deciding on the language of therapy. These factors are discussed below. 
(i) The age of the client and (planned) language of tuition. If the bilingual client is very young (e.g., if the client is a two year old, language-delayed boy), age will not be as important a consideration as when the client is older and at school or will attend school shortly. The reason for this is that younger clients have a greater chance of becoming proficient in both languages (no matter which one receives focus first in therapy) before school-going age than older clients have. For the older client, the clinician might suggest that the language of tuition, or planned tuition ${ }^{28}$, is used during therapy ${ }^{29}$.

(ii) The client's other disabilities. If the client has other disabilities, such as hearing impairment or cerebral palsy, and will have special educational needs, the family should take the language of tuition at schools appropriate for the client into consideration, even if the client is still very young ${ }^{30}$. In the case of bilingual children with disabilities, Toppelberg, Snow, and Tager-Flusberg (1999:1198-1199) propose the following provisional guidelines:

(a) Determine (1) the critical communicative needs of the client, (2) with which languages the client absolutely must be familiar, (3) the client's communicative abilities in the first and the second language, (4) the willingness and ability of family members and school personnel to function in the various possible languages, and (5) the client's attitude toward and aptitude for language in general and learning a second language in particular.

(b) Involve the family in making an informed decision regarding language of tuition. The clinician and family should weigh the importance of maintaining the home language against other, competing needs (e.g., the planned language of tuition).

(c) Do not encourage caregivers to speak the client's second language at home if they are not proficient in this language. Optimal, intense, well-structured, and first language-like input should be provided in the language(s) that it has been determined the client should be familiar with.

(iii) The family's future residential arrangements. If, in the near future, the client will move away from the geographical region in which he is currently living to a region with a different language profile, this should be kept in mind when selecting the language of therapy. 
(iv) The main caregiver's first language. The language spoken to the client by the main caregiver, i.e., the person who spends the most time communicating with the client, might be chosen as therapy language, even if this language is not the parents' first language, provided that this language is at least one of the parents' languages ${ }^{31}$.

(v) The strength and status of family relationships. When making choices for language of therapy based on the language of one of the caregivers, it should be borne in mind that child-care arrangements might change when caregivers' relationships change.

(vi) The dominant language of the client's siblings. If the caregivers are bilingual but the client's siblings are not, or are not bilingual to the same extent as the caregivers, the (dominant) language of the client's siblings should be the language of preference when choosing the therapy language.

(vii) Caregiver preferences. According to Ara and Thompson (1989), the choice of language to be used during therapy should, in the last instance, rest with the client's family $^{32}$. Whereas it is the clinician's task to point out that all languages are linguistically equal, the clinician has to submit to the caregivers' final choice of language for therapy, even if this choice is based solely on preconceptions or social presumptions.

(viii) The clinician's competence in providing therapy in a particular language. The only factor that can override the caregivers' preference is the clinician's competence in providing effective therapy in the language that the caregivers choose. If a clinician is not competent in the language that would suit the caregivers, or the client's needs, best, and if no other therapy providers or suitable interpreters are available, then the clinician should provide therapy in the language(s) in which she is competent to do so.

As Miller (1984a:183) states, "It is not the task of the therapist to alter religious or cultural values, but it is their task, as exhaustively and as sympathetically as possible, to explain and discuss with families the full range of possibilities and the long- and short-term implications of any decision", including decisions on language of therapy. 


\subsection{Parent guidance}

What is mostly referred to as "parent guidance" should be renamed "caregiver guidance", because the person attending therapy with the client is not necessarily one of the parents. In some cultures, there may be more than one caregiver, with none being (easily) identifiable as the main caregiver (Miller 1984a:174-175), and this is the case whether the client is bilingual or not. The middle-class, Western models of a central mother-child relationship and a physically present father may not apply to all language and cultural groups. The clinician needs to be aware of this, and be prepared for various people attending caregiver guidance sessions. This means that ground covered in previous sessions with another caregiver may have to be covered again with a different caregiver, often without prior warning.

Therapy practices aimed at training caregivers to interact with the client in ways that will promote language development are vulnerable to cultural bias (Van Kleeck 1994:68). Although most of these practices are based on research on parent-child interaction, the majority of the subjects in such research studies belong to white, middle-class, American or British families. Language use is essentially a cultural phenomenon which reflects and transmits cultural values and beliefs about, among others, the value of talk, the handling of status in interaction, teaching language to children, social organisation related to interaction, and about intentionality (Van Kleeck 1994:68-74). In order to provide sensible guidance to caregivers, the clinician needs to be aware that no assumptions can be made about any particular family.

One topic that needs to be addressed during caregiver guidance is that of choice of language in the home. Caregivers are often interested in obtaining information on how best to raise their children bilingually ${ }^{33}$, and, in some cases, how best to raise their children in English, even though the caregivers' proficiency in English may not be equal to that of a first language speaker. Despite the social, educational, and political reasons the caregivers may offer for their choice of English as medium of communication with the client, clinicians should take care to inform caregivers of the probable consequence of using English as the only language at home if English is the client's weaker language, namely that of subtractive bilingualism ${ }^{34}$ (Gutierrez-Clellen 1999:291). Furthermore, caregivers need to know that their communication 
strategies (including the choice of the language in which they address the child) are decisive for establishing active bilingualism (as opposed to passive bilingualism, where the child understands both languages spoken at home but chooses to speak mainly one), and thus for preserving the child's "other" (read: not English) language (Kasuya 1998; Lanza 1997).

\subsection{Therapy in the school setting}

According to Cummins (1984:136), the failure of school personnel to distinguish between the development of conversational or surface fluency (i.e., BICS) in a language, on the one hand, and CALP in that language, on the other hand, can result in poor academic performance being attributed to deficient cognitive or personality traits within minority-language students. The clinician has to be aware of the difference between BICS and CALP, and between additive and subtractive bilingualism, as well as of the language policy of the school in which she provides therapy. School personnel may often refer students with adequate BICS but poor CALP, as well as students with normal second languagelearning difficulties, to the clinician for speech-language therapy. The clinician has the responsibility to educate school personnel on what is and is not classified as a language impairment. Clinicians should take care not to act unwittingly as second languageteachers when they have been employed as speechlanguage therapists.

\section{MAKING USE OF INTERPRETERS}

Where the client is bilingual but the caregiver, or other accompanying adult, is monolingual and speaks a language that the clinician does not, an interpreter should be used. When choosing an interpreter and while using the services of one, caution should be exercised to avoid miscommunication and offense. In this section, some guidelines for selecting interpreters are given.

It should be borne in mind that the clinician is looking for more than a translator. She requires someone who can translate and interpret, reinforce the client's correct responses, encourage the client, and monitor the client's state of mind and physical well-being during therapy sessions. This said, the clinician may have to settle for the expertise of an accurate translator. 
Therefore, someone who has limited ability in either the caregiver's or the clinician's language should not be used (Barnett 1989:102; Chamberlain and Medinos-Landurand 1991:137).

Even someone who is fully bilingual may not always make a good interpreter. A good interpreter needs to be able to "transmit exactly what a person has requested, and likewise to reply" (Miller 1984a:192). A good interpreter, furthermore, is able to listen to and understand what the purpose of the interview, therapy activity, or counselling session is; to request and provide only what was originally requested or provided ${ }^{35}$ (i.e., not "colouring" the requests or responses with paraphrasing or own value judgements, not adding to or subtracting from responses, not interpreting the responses, and not filtering replies); and to treat the client with the same amount of respect shown by the clinician ${ }^{36}$. Apart from criteria regarding communication abilities and interpersonal skills, it may be necessary, in some situations, to consider the following when selecting an interpreter: (i) gender, as clients from certain cultures may find it difficult to discuss intimate issues in the presence of someone of the opposite sex; (ii) culture, because beliefs regarding the causes of medical conditions differ among cultures ${ }^{37}$; and (iii) age, in order to avoid what Barnett (1989:102) terms "intergenerational conflict" on matters such as child-rearing practices. An interpreter must also be aware of issues of confidentiality (Chamberlain and Medinos-Landurand 1991:137; Erickson and Iglesias 1986:207).

Interpreters who meet all or most of the above-mentioned criteria may not always be available; in fact, in South Africa, the availability of such interpreters to clinicians is extremely limited. Jordaan and Yelland (2003:25) found that $28 \%$ of the 25 clinicians who took part in their survey had interpreters available to assist during language therapy and that all of these clinicians who had access to interpreters worked in a hospital clinic. Where interpreters were not available, the clinicians in the Jordaan and Yelland study made use of parents to communicate with their child clients. Bearing in mind the qualities and skills required of a good interpreter, this practice is not recommended; however, currently, few (if any) alternatives exist for South African clinicians. 


\section{CONCLUSION}

Attitudes to languages and language varieties influence views on how and where the languages and language varieties should be used and taught, and many problems are created by misguided political-educational decisions, however well-intentioned (Miller 1984d:103). Therefore, clinicians need to be aware of their attitudes to bilingualism and bilingual communities, and of the basis for these attitudes.

This article has not offered many concrete solutions to the challenges faced by the clinician when managing bilingual clients. The reason for this is that, at the moment, there are, unfortunately, not many solutions. Even in the absence of such solutions to the challenge of fair diagnosis and treatment of possible language impairment in bilingual children, clinicians need to be aware of this challenge and of their own prejudices, assumptions, and preconceived ideas about bilinguals and bilingual communities. The informed clinician will be better able to judge which language difficulties are centred on the client's own development (and which of these exist due to language impairment versus due to normal second languagelearning processes), and which language difficulties are products of having to communicate in a society biased against the client. 


\section{NOTES}

1 Throughout this article, "bilingualism" is used as a cover term, which refers to bilingualism, multilingualism, and bidialectism. No clear distinction will be made between bilingualism, on the one hand, and biculturalism or multiculturalism, on the other. In the literature on bilingualism, bilinguals are often referred to as "non-English proficient speakers" or "minority speakers". In South Africa, the latter two terms are not as applicable as they are in certain other parts of the world (e.g., the United States of America or Britain), because, in South Africa, one of the bilingual's languages is not necessarily English, and neither of the bilingual's languages is necessarily a minority language. In this article, then, when the term "bilingual" is used, it may refer to a speaker of, for example, Xhosa and Sotho, Tswana and English, or standard South African English and so-called "Cape Flats English".

2 In a survey of 25 South African speech-language therapists, Jordaan and Yelland (2003:23-25) found that $88 \%$ of the clinicians provided therapy in only one of the client's languages (20\% in the client's first language and $68 \%$ in the client's second language). Only $12 \%$ of the clinicians provided therapy to their clients in both the clients' languages.

3 People who became bilingual during their childhood by no means form a homogeneous group. For an introduction to language acquisition in bilingual children, see Kessler (1984).

4 See Valdés and Figueroa (1994:30-34) for a discussion on what it means to know a language.

5 The practice of not asking questions regarding the home environment, child-rearing practices, and attitudes toward disabilities from clients who share the clinician's language and cultural background is not condoned. It goes without saying that the clinician should not assume any similarity between her environments, practices, and attitudes and those of her clients simply because they have the same language and/or cultural background.

6 See Heath (1983) for an account of normal child language acquisition and the differences in the child-rearing practices in two North American communities. Although this book does not deal with language assessment or therapy, it gives an excellent overview of sociolinguistic influences on child language development, and it may challenge some ideas that clinicians have on what constitutes normal caregiver-child interaction.

7 Taylor (1986:13-14) states,

With respect to the standard list of causative factors associated with communication pathology, the point must be reiterated that although the causative factors ... probably exist all over the world, cultural factors influence the value and perceptions that interact with them. For example, a poor diet in one culture may be considered to be a good diet in another culture. Likewise, one culture's view of a satisfactory social environment may be another culture's perception of a completely unacceptable social environment. 
8 The issues mentioned in this paragraph apply to clients of all cultures. The clinician should, however, bear in mind that matters that cause shame or constitute a threat to face differ among cultures.

9 See Mbali (1995) for examples of intercultural interpersonal communication, including case-history-taking, in the medical setting.

10 See Mesthrie (1994:187-190) for a discussion on tag questions in South African Black English.

11 See Hall (1995:77) for an example of a basic language usage profile.

12 These advantages include, among others, ease of administration, the ability to compare the client's performance to that of his peers, and the possibility of re-administration to measure progress made during therapy.

13 See Wolfram (1983) for a brief discussion of the general arguments against the use of formal assessment instruments, and see Damico (1991:171-177) for a brief discussion of the problematic characteristics of the current assessment approach (that of using standardised, discrete-point assessment instruments). These characteristics include lack of linguistic realism, lack of authenticity, poor psychometric strength, and inherent and unavoidable bias toward the culture for which the assessment instruments were developed. Also see Oller (1983) for a discussion of the following characteristics of language that complicate traditional language assessment: (i) language is symbolic and creative in nature, which makes for difficult formal assessment; (ii) language is synergistic, and a measure of a part thereof is not necessarily an accurate reflection of the whole; (iii) language forms a part of the child's total world of experience, and it is difficult to isolate language in order to assess it separately from the child's other abilities.

See Saville-Troike (1986:50-52) for a very informative example of this point.

15 See Pakendorf and Alant (1997) for a description of the process by which the Northern Sotho translation of the Peabody Picture Vocabulary Test - Revised was made culturally more valid.

16 For example, if one of the items is a picture of a bed-side lamp, decide whether a four year old client from a rural township without electricity would be sufficiently familiar with bed-side lamps to warrant inclusion of this item.

17 The client may, for instance, point to a picture of a basketball hoop, but name the object depicted correctly as "net".

18 For example, when showing pictures of a carrot, a patato, and an apple, and asking the client to point to the two that go together, he may point to the carrot and the apple, because that is what he eats during break-time at school, instead of pointing to the two vegetables.

19 Miller (1984a:174) provides examples of this normal variation. 
See Gutiérrez-Clellen, Restrepo, Bedore, Peña, and Anderson (2000) for a discussion on similar work on the assessment of American English-Spanish bilinguals.

21 Input in the first language develops conceptual-linguistic knowledge, or cognitive/academic language proficiency (CALP), which enables the learner to manipulate successfully the language encountered in decontextualised academic situations (Cummins 1979). CALP is distinguished from so-called "surface fluency", or basic interpersonal communication skills (BICS) and defined as "the manifestation of language proficiency in everyday communicative contexts". According to Cummins (1981:41),

Minority-language children [non-first language speakers of English - FS] whose L1 [= first language - author] proficiency on starting school is well-developed [i.e., children who possess CALP in their first language - FS] will usually have little difficulty acquiring high levels of English proficiency. However, children whose overall conceptual abilities [or CALP - FS] in both L1 and L2 [= second language - FS] are poorly developed are likely to experience difficulty in school.

22 When discussing the identification of learning disabilities in minority students, Cummins (1984:86) states that

if the consequences of incorrect identification were simply that children received extra help that they did not need, then there might be relatively little concern about overinclusion of children in special education classes. However, the effects that labels exert on teachers' expectations and on children's academic self-image have been repeatedly documented.

Vaughn-Cooke wrote specifically with the minority African-American population in mind, but these proposals are applicable to all assessment situations where the client is of a language and/or cultural group that is underrepresented in test design.

24 See Damico and Oller (1980) and Oller (1983:80-85) for early references to such pragmatic criteria.

25 Rollins et al. (2000:226) state that, in general, children tend to tell their best stories about a personal experience of pain or fear. The authors provide examples of story prompts that can be used by clinicians to elicit stories from child clients on these topics, such as: "I was sweeping the porch the other day and picked up a flower pot and there was a bee underneath and it stung me. Did anything like that ever happen to you?"

26 For other studies with similar results, see Garcia (1983), Kiernan and Swisher (1990), and Perozzi (1985).

27 Cummins (1984:144) mentions some of the literacy-related proficiencies underlying both languages:

Conceptual knowledge is perhaps the most obvious example. ... subject matter knowledge, higher order thinking skills, reading strategies, writing compositions skills, etc. developed through the medium of L1 transfer or become available to L2 given sufficient exposure and motivation. 
28 For a discussion on general trends in choice of language of tuition in Africa (i.e., choosing between African languages and English, French, or Portuguese), see Roy-Campbell (2003).

29 The clinician should discuss with the caregiver the possibility that children who are insecure about the value of their culture often perform poorly in a school in which the medium of instruction is not the child's first language. See Cummins (1981:19).

30 For example, if the Afrikaans- and Tswana-speaking language-impaired client has a hearing disability, the caregivers might decide to choose the language of therapy based on the availability of Afrikaans and Tswana facilities for hearing-impaired children in their geographical area.

31 This factor is mostly a consideration when the main caregiver belongs to the same (extended) family or cultural group as the parents.

32 Note, however, that, according to Clause 29 of the 1996 South African Bill of Rights, this choice seems to rest with the child and not the caregivers: "Everyone has the right to receive education in the official language or languages of their choice in public educational institutions where that right is reasonably practicable. ..." (emphasis added).

33 Written guides are available for caregivers interested in advice on raising children bilingually, e.g. Arnberg (1987), Baker (1995), Cunningham-Andersson and Andersson (1999), and Harding and Riley (1986). It goes without saying that the advice given reflects the authors' theoretical stances on issues pertaining to childhood bilingualism. For this reason, it is recommended that clinicians screen these guides before recommending their use to caregivers.

34 "Subtractive bilingualism" refers to the type of bilingualism in which a person begins to lose his/her first language before having developed age-appropriate proficiency in his/her second language.

35 Cases of interpreters interpreting literally, rather than translating accurately, the clinician's questions and given information abound. For example, Miller (1984a:192-193) reports that, at one point during an assessment session, a thoroughly-instructed interpreter took unduly long to ask a question. It later came to light that the interpreter asked the client to point to the picture of the bath, as requested by the clinician. When the client did not respond, the interpreter, suspecting that the client knew what a bath looked like, asked the client to point to the picture next to that of the chair.

36 The clinician should be aware of possible miscommunication and of the added difficulties of establishing rapport with the client through an interpreter (Barnett 1989:104).

37 See Mbali (1995) for examples drawn from Southern African cultures. 


\section{REFERENCES}

Ara, F. and C. Thompson. 1989. Intervention with bilingual pre-school children. In D.M. Duncan (ed.) Working with bilingual language disability. New York: Chapman and Hall. pp. 132-153.

Arnberg, L. 1987. Raising children bilingually: The pre-school years. Clevedon: Multilingual Matters.

Baker, C. 1995. A parents' and teachers' guide to bilingualism. Clevedon: Multilingual Matters.

Barnett, S. 1989. Working with interpreters. In D.M. Duncan (ed.) Working with bilingual language disability. New York: Chapman and Hall. pp. 91-112.

Chamberlain, P. and P. Medinos-Landurand. 1991. Practical considerations for the assessment of LEP students with special needs. In E.V. Hamayan and J.S. Damico (eds.) Limiting bias in the assessment of bilingual students. Austin: Pro-Ed. pp. 112-156.

Craig, H.K. and J.A. Washington. 1994. The complex syntax of poor, urban, African American preschoolers at school entry. Language, Speech, and Hearing Services in Schools 25: 181-190.

Craig, H.K. and J.A. Washington. 2000. An assessment battery for identifying language impairments in African American children. Journal of Speech, Language, and Hearing Research 43: 366-379.

Craig, H.K., J.A. Washington, and C. Thompson-Porter. 1998a. Performances of young African American children on two comprehension tasks. Journal of Speech, Language, and Hearing Research 41: 445-457.

Craig, H.K., J.A. Washington, and C. Thompson-Porter. 1998b. Average C-unit lengths in the discourse of African American children from low-income, urban homes. Journal of Speech, Language, and Hearing Research 41: 433-444.

Cummins, J. 1979. Cognitive/academic language proficiency, linguistic interdependence, the optimum age question and some other matters. Working Papers on Bilingualism 19: 121-129.

Cummins, J. 1981. Bilingualism and minority-language children. Ontario: The Ontario Institute for Studies in Education. 
Cummins, J. 1984. Bilingualism and special education: Issues in assessment and pedagogy. San Diego: College-Hill Press.

Cummins, J. 1989. Empowering minority students. Sacramento: California Association for Bilingual Education.

Cunningham-Andersson, U. and S. Andersson. 1999. Growing up with two languages: A practical guide. London: Routledge.

Damico, J.S. 1991. Descriptive assessment of communicative ability in limited English proficient students. In E.V. Hamayan and J.S. Damico (eds.) Limiting bias in the assessment of bilingual students. Austin: Pro-Ed. pp. 158-217.

Damico, J. and J. Oller. 1980. Pragmatic versus morphological/syntactic criteria for language referrals. Language, Speech, and Hearing Services in Schools 11: 85-94.

De Villiers, P.A., J.G. De Villiers, T. Roeper, H.N. Seymour, and B. Zurer Pearson. 2003. Unbiased assessment of first language acquisition in English: Distinguishing development and dialect from disorder. Stellenbosch Papers in Linguisitics Plus 32: 99-121.

Erickson, J.G. and A. Iglesias. 1986. Assessment of communication disorders in non-English proficient children. In O.L. Taylor (ed.) Nature of communication disorders in culturally and linguistically diverse populations. San Diego: College-Hill Press. pp. 181-217.

Garcia, E. 1983. Early child bilingualism with special reference to the Mexican-American child. Albuquerque: University of New Mexico Press.

Grosjean, F. 1989. Neurolinguists, beware! The bilingual is not two monolinguals in one person. Brain and Language 36: 3-15.

Gutierrez-Clellen, V.F. 1999. Language choice in intervention with bilingual children. American Journal of Speech-Language Pathology 8: 291-302.

Gutiérrez-Clellen, V.F., M.A. Restrepo, L. Bedore, A. Peña, and R. Anderson. 2000. Language sample analysis in Spanish-speaking children: Methodological considerations. Language, Speech, and Hearing Services in Schools 31: 88-98.

Hall, D. 1995. Assessing the needs of bilingual pupils. London: David Fulton Publishers.

Harding, E. and P. Riley. 1986. The bilingual family: A handbook for parents. Cambridge, UK: CUP. 
Harris, G.A. 1986. Barriers to the delivery of speech, language, and hearing services to native Americans. In O.L. Taylor (ed.) Nature of communication disorders in culturally and linguistically diverse populations. San Diego: College-Hill Press. pp. 219-236.

Heath, S.B. 1983. Ways with words: Language, life, and work in communities and classrooms. Cambridge, UK: CUP.

Jordaan, H. 1996. Principles and procedures for the assessment of language proficiency of bilingual children within the South African context: Some preliminary data. Stellenbosch Papers in Linguistics Plus 29: 339-359.

Jordaan, H. and A. Yelland. 2003. Intervention with multilingual language impaired children by South African speech-language therapists. Journal of Multilingual Communication Disorders 1(1): 13-33.

Kasuya, H. 1998. Determinants of language choice in bilingual children: The role of input. International Journal of Bilingualism 2(3): 327-346.

Kessler, C. 1984. Language acquisition in bilingual children. In N. Miller (ed.) Bilingualism and language disability. San Diego: College Hill Press. pp. 26-55.

Kiernan, B. and L. Swisher. 1990. The initial learning of novel English words: Two singlesubject experiments with minority-language children. Journal of Speech and Hearing Research 33: 707-716.

Langdon, H.W. 1989. Language disorder or difference? Assessing the language skills of Hispanic children. Exceptional Children 56(2): 160-167.

Lanza, E. 1997. Language mixing in infant bilingualism. Oxford: OUP.

Mbali, C. 1995. Multilingual medical communication. The challenges for curriculum development. Constitutionally enshrined multilingualism: Challenges and responses. Proceedings of the $15^{\text {th }}$ Annual Conference of the Southern African Applied Linguistics Association, Stellenbosch, 10-12 July 1995. pp. 555-574.

Mesthrie, R. 1994. Standardisation and variation in South African English. Stellenbosch Papers in Linguistics Plus 26: 181-201.

Miller, N. 1984a. The case history in a cross-cultural milieu. In N. Miller (ed.) Bilingualism and language disability. San Diego: College Hill Press. pp. 169-176.

Miller, N. 1984b. Language use in bilingual communities. In N. Miller (ed.) Bilingualism and language disability. San Diego: College Hill Press. pp. 3-25. 
Miller, N. 1984c. Some observations concerning formal tests in cross-cultural settings. In N. Miller (ed.) Bilingualism and language disability. San Diego: College Hill Press. pp. 107-114.

Miller, N. 1984d. Language problems and bilingual children. In N. Miller (ed.) Bilingualism and language disability. San Diego: College Hill Press. pp. 81-103.

Miller, N. and S. Abudarham. 1984. Management of communication problems in bilingual children. In N. Miller (ed.) Bilingualism and language disability. San Diego: College Hill Press. pp. 177-198.

Müller, N. 2003. Multilingual communication disorders. Journal of Multilingual Communication Disorders 1(1): 1-12.

Oller, J.W., Jr. 1983. Testing proficiencies and diagnosing language disorders in bilingual children. In D.R. Omark and J.G. Erickson (eds.) The bilingual exceptional child. San Diego: College-Hill Press. pp. 71-87.

Pakendorf, C. and E. Alant. 1997. Culturally valid assessment tools: Northern Sotho translation of the Peabody Picture Vocabulary Test-Revised. The South African Journal of Communication Disorders 44: 3-12.

Peña, E.D. and R. Quinn. 1997. Task familiarity: Effect on the test performance of Puerto Rican and African American children. Language, Speech, and Hearing Services in Schools 28: 323-332.

Peña, E., R. Quinn, and A. Iglesias. 1992. The application of dynamic methods to language assessment: A nonbiased procedure. The Journal of Special Education 26: 269-280.

Perozzi, J.A. 1985. A pilot study of language facilitation for bilingual, language-handicapped children: Theoretical and intervention implications. Journal of Speech and Hearing Disorders 50: 403-406.

Perozzi, J.A. and M.L.C. Sanchez. 1992. The effect of instruction in L1 on receptive acquisition of L2 for bilingual children with language delay. Language, Speech, and Hearing Services in Schools 23: 348-352.

Rollins, P. R., A. McCabe, and L. Bliss. 2000. Culturally sensitive assessment of narrative in children. Seminars in Speech and Language 21: 223-234.

Roy-Campbell, Z.M. 2003. Promoting African languages as conveyors of knowledge in educational institutions. In S. Makoni, G. Smitherman, A.F. Ball, and A.K. Spears 
(eds.) Black linguistics. Language, society, and politics in Africa and the Americas. London and New York: Routlegde. pp. 83-102.

Saville-Troike, M. 1986. Anthropological considerations in the study of communication. In O.L. Taylor (ed.) Nature of communication disorders in culturally and linguistically diverse populations. San Diego: College-Hill Press. pp. 47-72.

Schreier, A.R. 1991. Communication. In M.A. Kibel and L.A. Wagstaff (eds.) Child health for all. Cape Town: OUP. pp. 349-353.

Seymour, H., T. Roeper, and J.G. de Villiers J.G. 2003a. Diagnostic Evalution of Language Variation (DELV): Screening Test. San Antonio: The Psychological Corporation, Harcourt Assessment Company.

Seymour, H., T. Roeper, and J.G. de Villiers J.G. 2003b. Diagnostic Evalution of Language Variation (DELV): Criterion Referenced. San Antonio: The Psychological Corporation, Harcourt Assessment Company.

Stobbart, C.L. 1992. Bilingualism: Theoretical perspectives on language diversity. The South African Journal of Communication Disorders 39: 13-23.

Stockman, I.J. 1986. Language acquisition in culturally diverse populations: The black child as a case study. In O.L. Taylor (ed.) Nature of communication disorders in culturally and linguistically diverse populations. San Diego: College-Hill Press. pp. 117-155.

Taylor, O.L. 1986. Historical perspectives and conceptual framework. In O.L. Taylor (ed.) Nature of communication disorders in culturally and linguistically diverse populations. San Diego: College-Hill Press. pp. 1-17.

Thordardottir, E.T., S.E. Weismer, and M.E. Smith. 1997. Vocabulary learning in bilingual and monolingual clinical intervention. Child Language Teaching and Therapy 13(3): 215-227.

Toppelberg, C.O., C.E. Snow, and H. Tager-Flusberg. 1999. Severe developmental disorders and bilingualism. Journal of American Child and Adolescent Psychiatry 38(9): 11971199.

Toronto, A.S. and S. Merrill. 1983. Developing local normed assessment instruments. In D.R. Omark and J.G. Erickson (eds.) The bilingual exceptional child. San Diego: College-Hill Press. pp. 105-121.

Valdés, G. and R.A. Figueroa. 1994. Bilingualism and testing: a special case of bias. Norwood: Abblex Publishing Corporation. 
Van Kleeck, A. 1994. Potential cultural bias in training parents as conversational partners with their children who have delays in language development. American Journal of Speech-Language Pathology 3: 67-78.

Vaughn-Cooke, B. 1983. Improving language assessment in minority children. ASHA 25(4): 29-34.

Washington, J. and H. Craig. 1998. Socioeconomic status and gender influences on children's dialectal variations. Journal of Speech, Language, and Hearing Research 41: 618-628.

Washington, J. and H. Craig. 1999. Performances of at-risk, African American preschoolers on the Peabody Picture Vocabulary Test-III. Language, Speech, and Hearing Services in Schools 30: 75-82.

Westby, C. 1990. Ethnographic interviewing: Asking the right questions to the right people in the right ways. Journal of Childhood Communicative Disorders 13(1): 101-111.

Wolfram, W. 1983. Test interpretation and sociolinguistic differences. Topics in Language Disorders 3(3): 21-34. 
